International Journal of Translation and Interpretation Studies (IJTIS)

ISSN: 2745-2602

DOI: $10.32996 /$ ijtis

Journal Homepage: www.al-kindipublisher.com/index.php/ijtis

\title{
Translating The Disaster of Mourice Blanchot Between Ann Smock and Azzedine Chentouf
}

\section{Dr. Soufiane Laachiri 8 (D)}

ترجمة فاجعة موريس بلانشو بين آن سموك وعز الدين الشنتوف

Researcher in cultural translation, orientalism and postcolonialism, Abdelmalek Essaàdi University, Morocco

$\triangle$ Corresponding Author: Dr. Soufiane Laachiri, E-mail: soufiane.laachiri@gmail.com

\section{ARTICLE INFORMATION}

Received: July 08, 2021

Accepted: August 25, 2021

Volume: 1

Issue: 1

DOI: 10.32996/ijtis.2021.1.1.3

\section{KEYWORDS}

Disaster, paradox, absolute impossibility, philosophical translation, obscurity and intended ambiguity

\section{ABSTRACT}

The present article attempts to present a succinct and circumspect comparison between two different translations for Mourice Blanchot's book « L'écriture du désastre ».The first translation was performed by Ann Smock in 1995 and was from French into English, while the other translation was skillfully produced by Azzedine Chentouf from French into Arabic in 2018. The contrast in attitudes and translational fertilization has provided us with ample opportunities to study, reflect on, and rethink the nexus of Blanchot's philosophy from different linguistic perspectives. However, in our attempt to formulate our judgments on the English and Arabic versions of the book, we can judge by an escapable logic and with analytical evidence that the English translation entitled «The writing of the disaster » has intensified the hold of a literal translation that makes the chances of being close to the original meaning of the source text depressingly small. Chentouf's translation, on the other hand, remains profoundly meaningful; it is capable of going down into the marrow of Blanchot's thought to assert understanding of his intellectual complexities. In brief, despite the triviality of the advanced examples, we are certain that Azzedine Chentouf, through his Arabic translation, knows the hard philosophical portrait of Mourice Blanchot in its inclusiveness. Therefore, it is no surprise that every choice he makes in this translation explains his tremendous efforts as a philosopher first before being ranked as a translator.

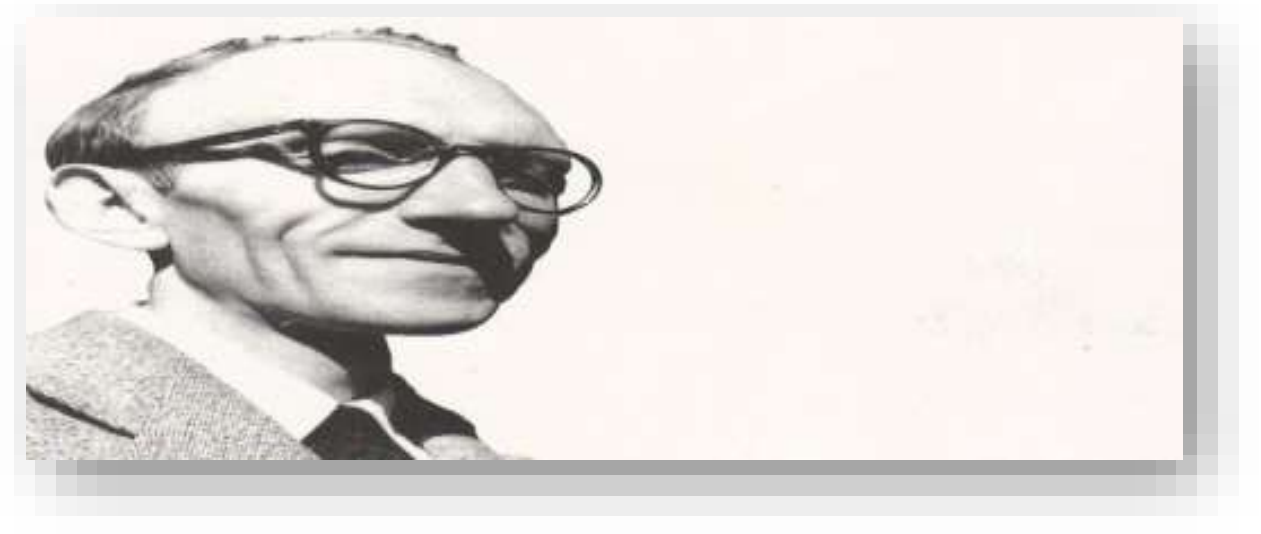

Mourice Blanchot (1907-2003)

$\mathrm{He}$ is a French writer, philosopher, and literary critic. He gained fame for his philosophical works, which had a strong influence on post-structuralist philosophers such as Gilles Deleuze, Michel Foucault, and Jacques Derrida. He spent most of his life between narration and philosophical investigation. His main works were primarily based on paradoxes and impossibilities, the most important of which is the present book we are dealing with, «l'écriture du désastre ». The latter has been translated many times into many languages but culminated in a skilful Arabic translation performed by a critic and a translator in the philosophical sense, Azzedine Chentouf. 
تقديم

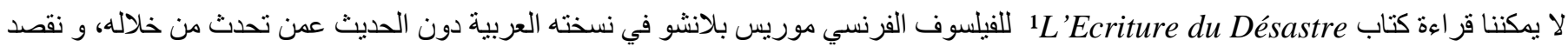

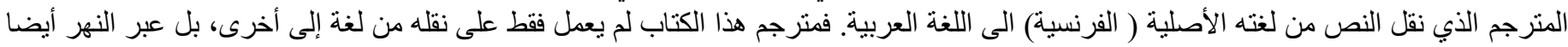

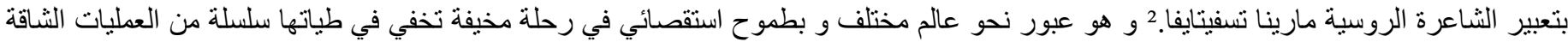

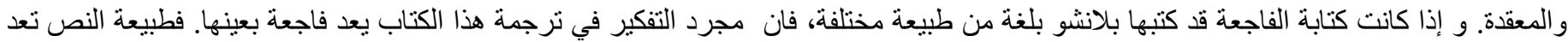

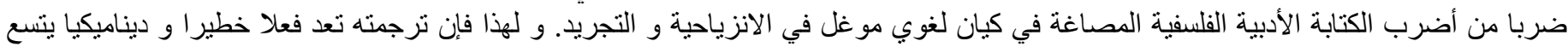

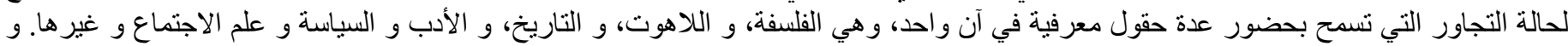

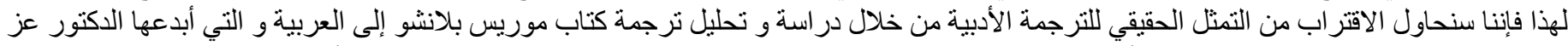
الدين الشنتوف3 في محاولة منقطعة النظير أسهمت في تعميق إدر الك فكر الفيلسوف بلانشو و فلك الاشتباك القائم بين نقاده و أفكاره.

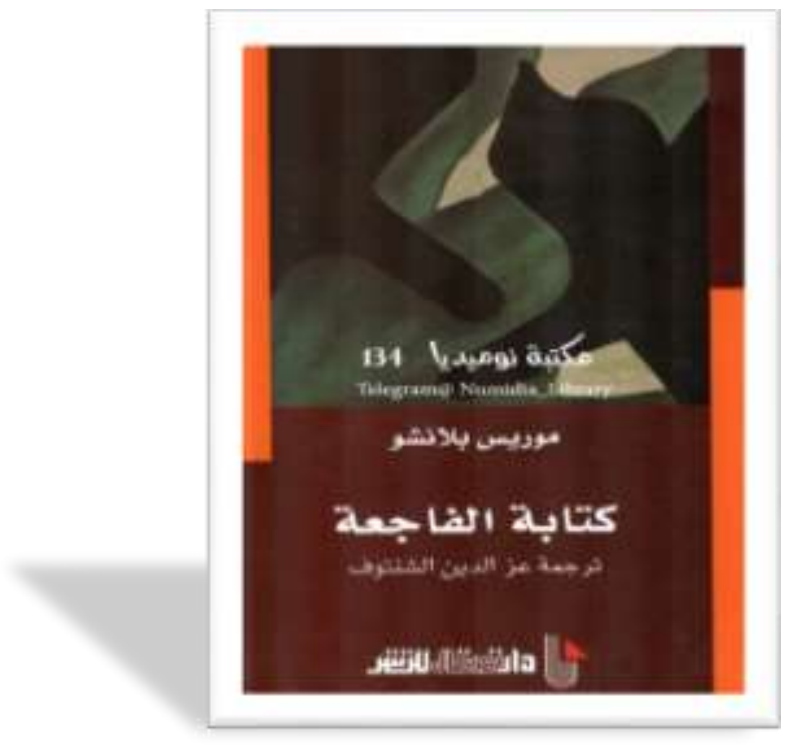

: مع الكتاب

عندما تقرأ عنوان الكتاب و تقر أ ما تحته لتجد بأنه مترجم إلى العربية، تعتريك مشاعر الدهشة و الانبهار لأن ترجمة هذا الفيلسوف بالذات و تلك النوعية

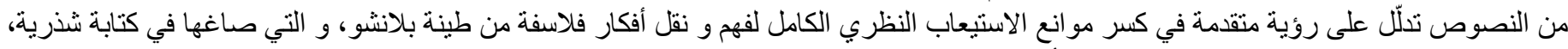

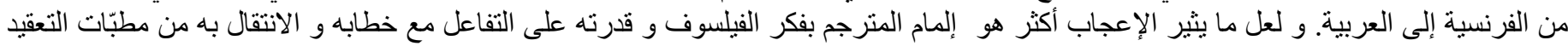
إلى سلاسة المعنى، حيث تحقى المعنى الحقيقي لمفهوم الترجمان الذي لا يكتفي بالنقل و لكن يتجاوز ذلك إلى التفسير و التأويل أيضا.

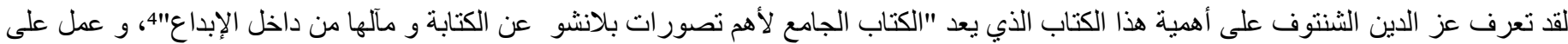

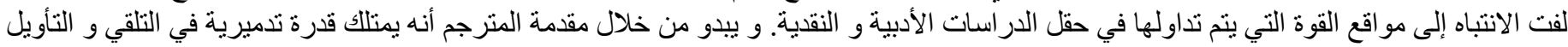

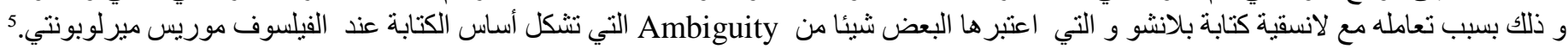

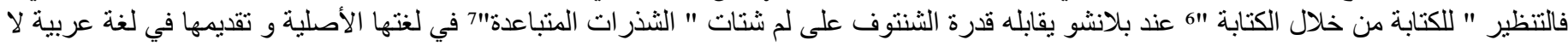

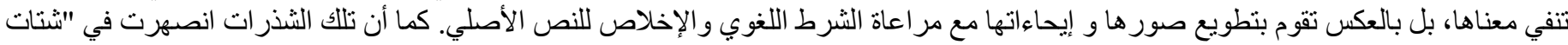

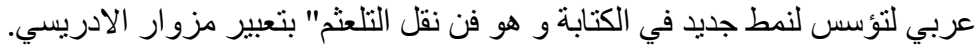
بين الفلسفة و الترجمة :

يرى طه عبد الرحمان بأن " الخصائص التجديدية للفلسفة "8 لا تتعارض مع الخصائص الأصلية للترجمة حيث إنهما نتنركان في "الخصوصية و القصدية

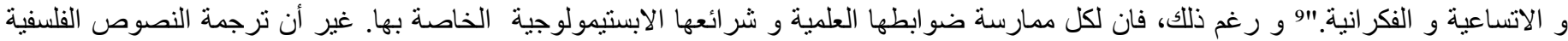

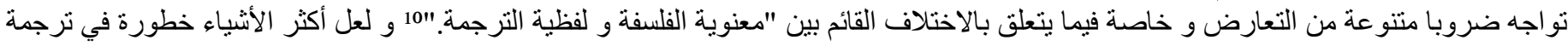

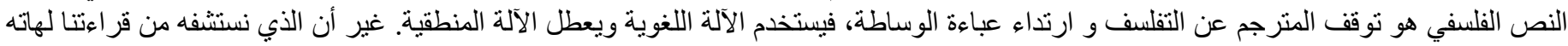

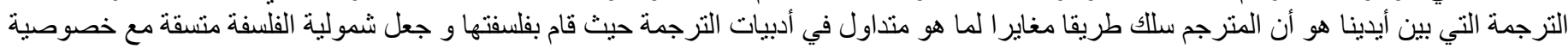
الترجمة. بين المترجم و الفيلسوف :

هنالك مقولة يتم ترديدها بين المترجمين و هي (الترجمة خيانة). 11 لكن هذه الفزّاعة لا يتم ترديدها أو الاستعانة بها إلا كلما تعلق الأمر بحقول معرفية مو غلة الته

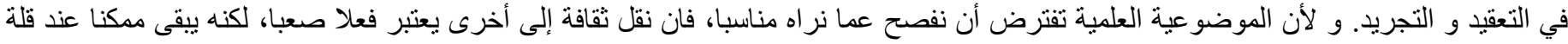

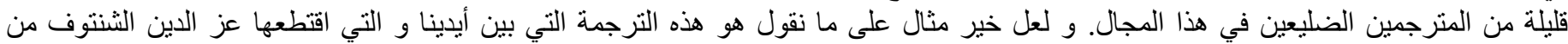


صيرورة الزمن و وضع أصبعه على ذروة اللحظة التي فكر فيها بلانشو. فجاءت ترجمته بإيقاع مباغت يصعب التمبيز بينها و بين كلام من كتب النص. و من هذا المنطلق يمكننا الجزم بأن ترجمة الثنتنوف تتم عن ثلاثة أمور متر ابطة :

أولها أن عز الدين الثنتوف تثبع بحس فلسفي عال حصنه من السقوط في عدم الفهم أو الانبهار بمنظومة أفكار بلانشو مهما كانت جاذبيتها. كما أنه لم يفسح

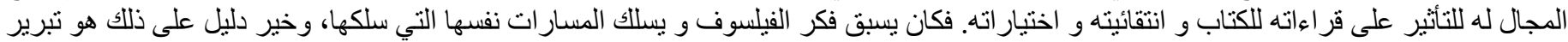

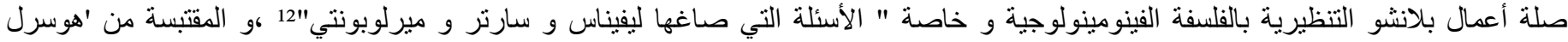

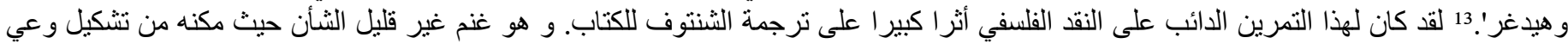

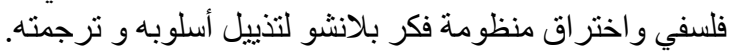

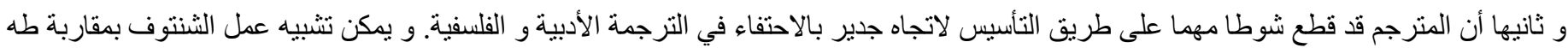

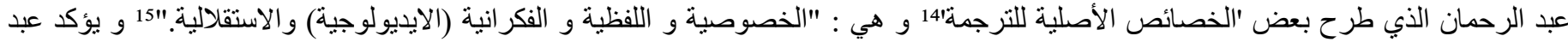

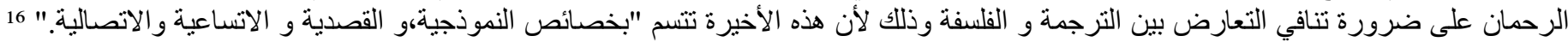

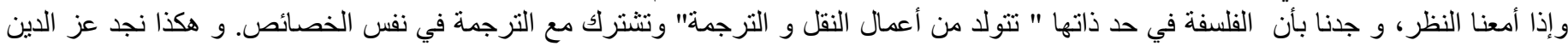

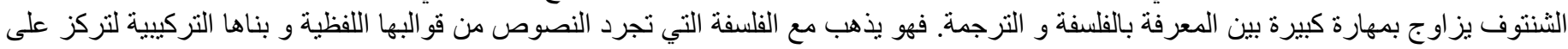

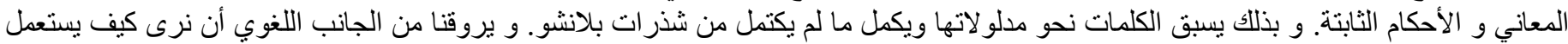

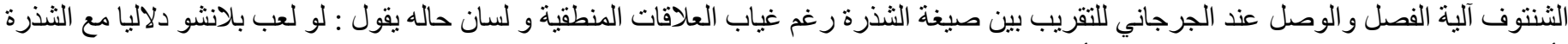

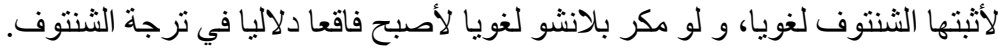

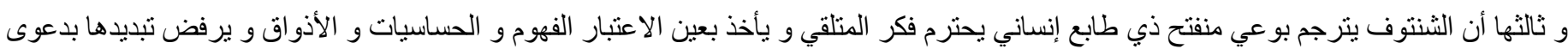

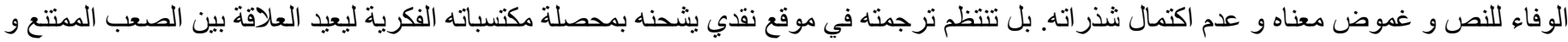

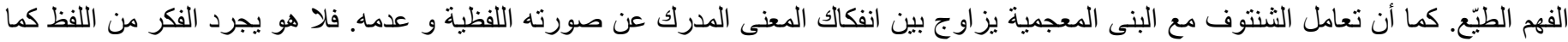

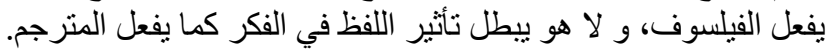

\section{على سبيل المقارنة :}

لقد كانت لبلانشو حظوة كبرى في الأوساط الأدبية المعاصرة كما يؤكد على ذلك عز الدين الثنتوف في مقدمة ترجمته. كما أن صيته في مجال الفلسفة

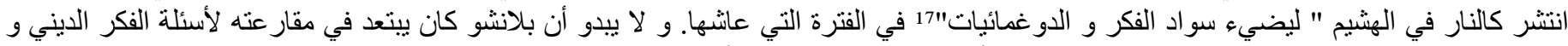

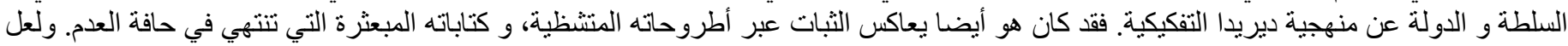

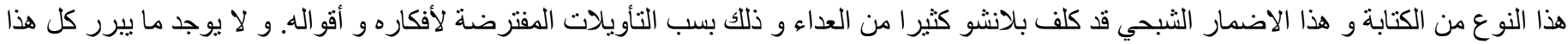

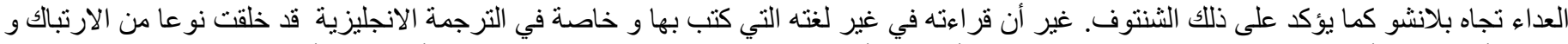

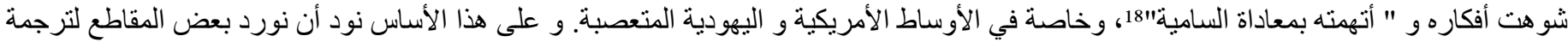
كتابة الفاجعة بالانجليزية و مقارنتها بالترجمة العربية لنكون قادرين على بناء مسافة نقدية مرتكزة على أسس مقارنة منينة. فلنأخذ المقاطع التالية على سبيل

Le désastre est séparé, ce qu'il y a de plus séparé.

Quand le désastre survient, il ne vient pas. Le désastre est son imminence, mais puisque le futur, tel que nous

le concevons dans l'ordre du temps vécu, appartient au désastre, le désastre l'a toujours déjà retiré ou dis-sùadé, il n'y a pas d'avenir pour le désastre, comme il n'y a pas de temps ni d'espace où il s'accomplisse. 
الترجمة الانجليزية20 - 20

Ann Smock

The disaster is separate ; that which is most separate.

When the disaster comes upon us, it doesn't come. The disaster is its imminence,but since the future, as we conceive of it in the order of lived time,belongs to the disaster, the disaster has always already withdrawn or dissuade it ; there is no future for the disaster, just as there is no time or space for its accomplishment.

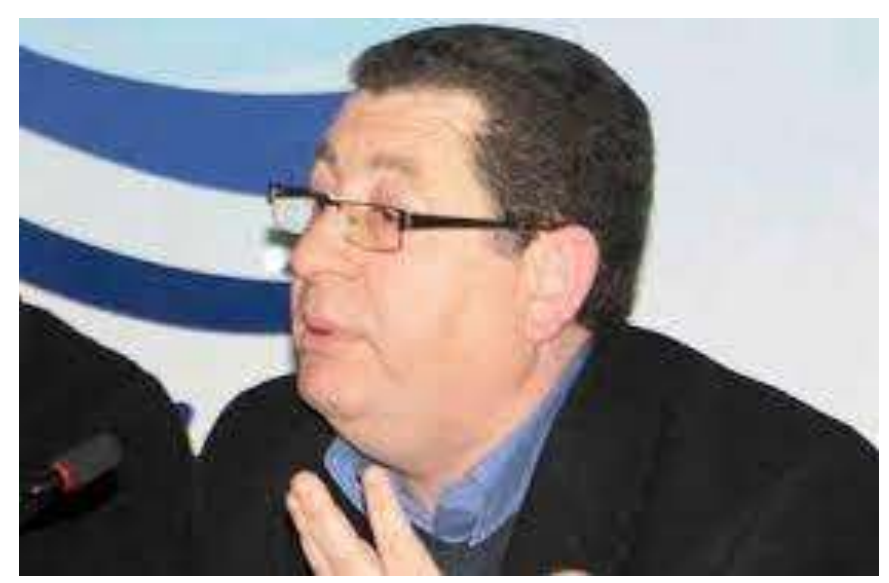

د. عز الدين الشنتوف

الفاجعة منعزلة، بل أكثر الأشياء انعز الا.

و عندما تباغت الفاجعة ،فإنها لا تأتي. و الفاجعة هي قدومها الوشيك، و بما أن المستقبل ، كما نتصوره ضمن نظام الزمن المعيش،ينتمي إلى لى

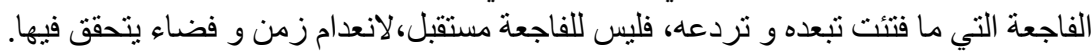

:تعليق :

ليس من السهل إبداء رأي في ترجمات لنص يتموج على اللانهائي و يرفض الثئي الثبات على حال واحد. إنه نص يجاوز المعنى الذي تحمله الألفاظ و ينفيها

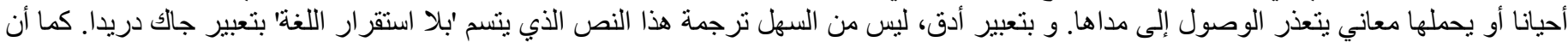

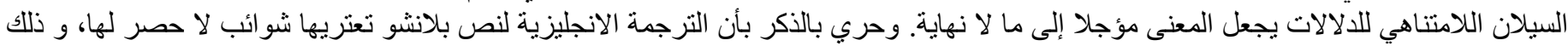

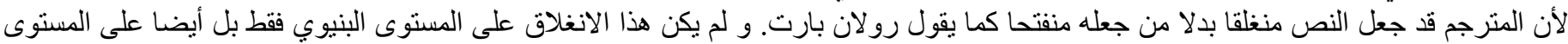
الدلالي. و لنأخذ الأمثلة التالية على سبيل المثال:

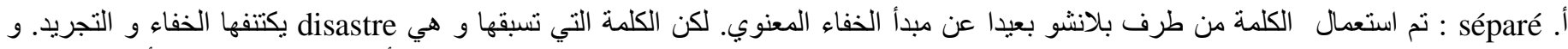

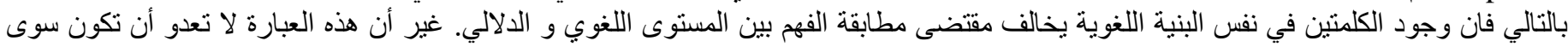

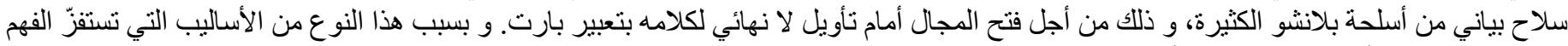

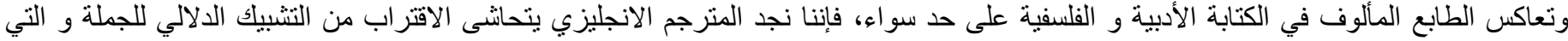
تحتمل عدة طيات و التواءوات تلغي أو تقلل من الفهم السلس لمقصدية بلانشو. فجاءت ترجمته بنزع حرفي و تكافؤ شكلي ينم عن علاقة ترجمية سطحية

مقتصدة شكلا و معنى.

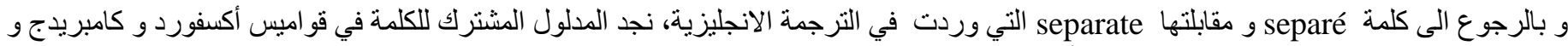

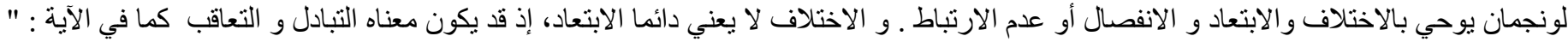

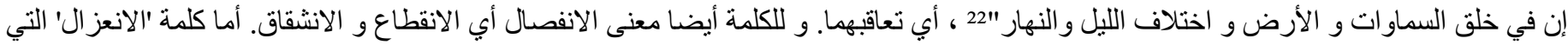

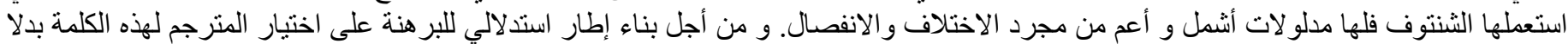

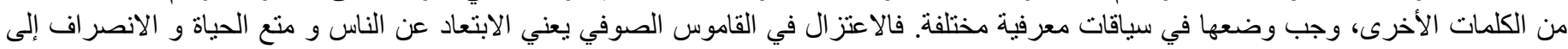

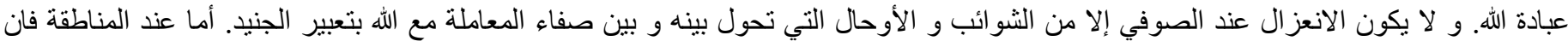




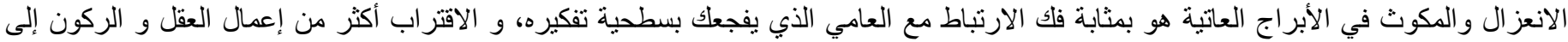

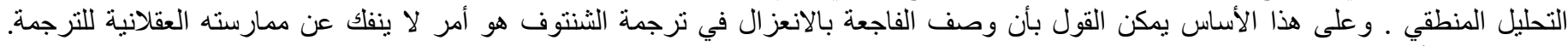

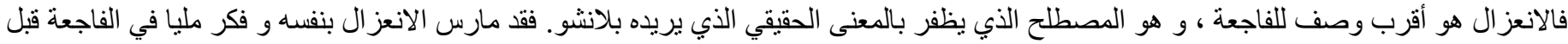

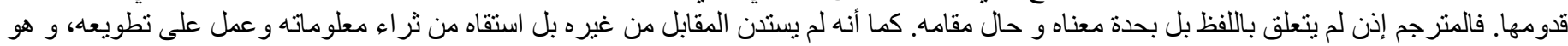

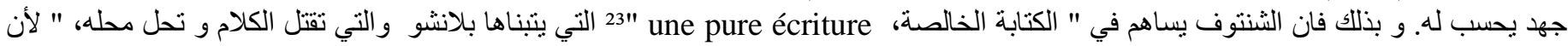
موت الكلام هو أفق اللغة وأصلها"24 كما يقول جالك ديريدا.

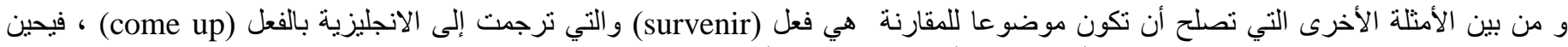

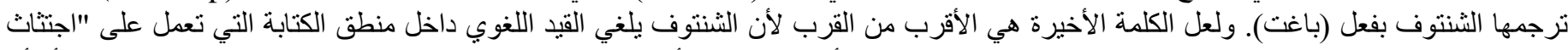

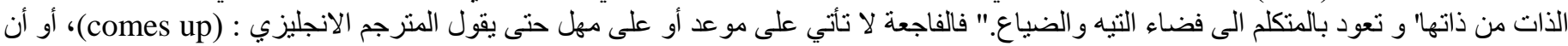

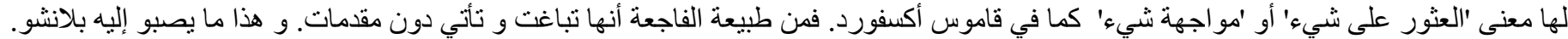

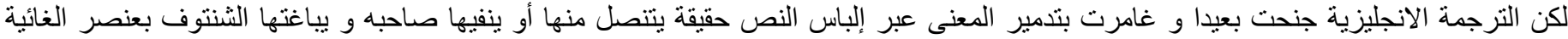

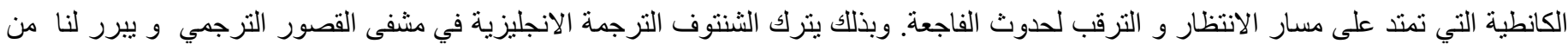

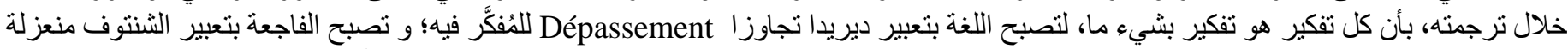

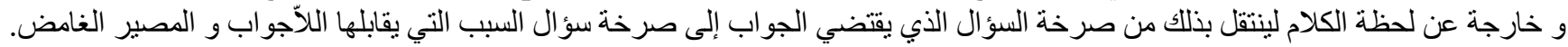
لنتذكر إذن بأن ترجمة الثنتوف تنم عن قدرة فائقة لانتقاء مصطلحات بكل عناية و تطويع المعنى. فقد حققت الكتابة عنده ما يتمناه المعنى دون أن يصرح

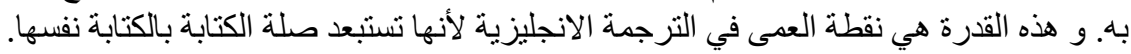

خلاصة :

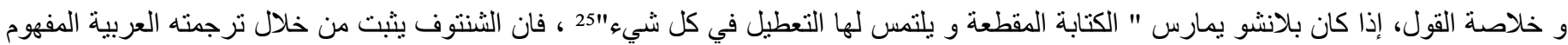

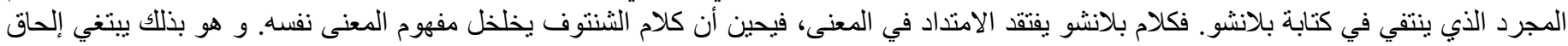

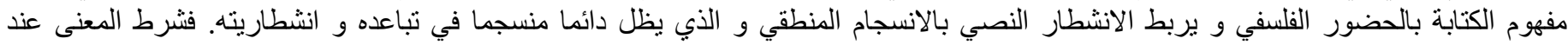

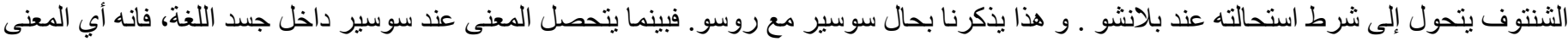

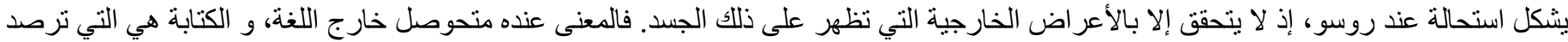
عيوب اللغة. و هل قال الثنتوف غير هذا؟ لألأ

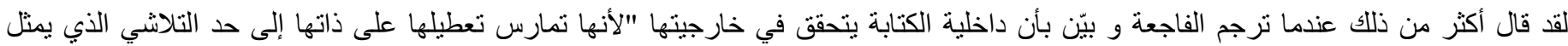

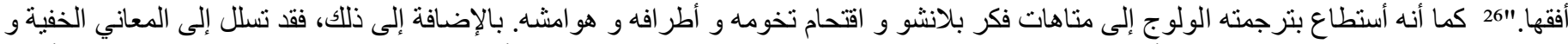

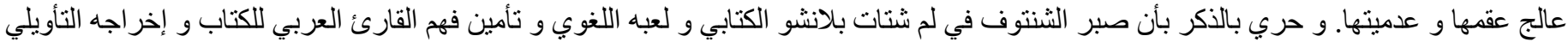

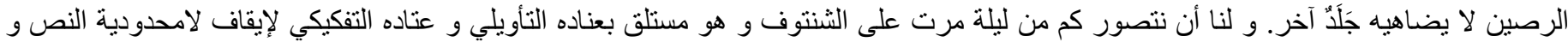

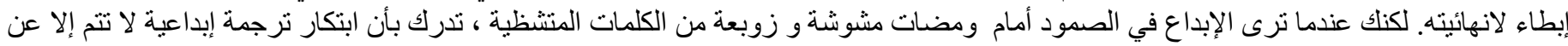

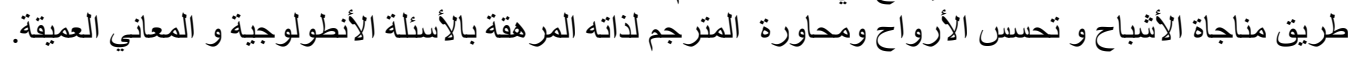
ترجمة الشنتوف اختلاف، و نمط غير معهود في النقل. استتطاق المبعثر و إعادة التشظيّ إلى أصله، قراعة جديدة لرنين بلانشو، و الأهم من كل ذللك، اكتثاف السر المجهول عند المترجم و الناقد الفذ عز الدين الشنتوف.

الهوامش :

كتابة الفاجعة، هو كتاب لمؤلفه الفيلسوف الفرنسي موريس بلانشو و الذي نشر سنة 1980 و ترجمه الى العربية الدكتور عز الدين الثنتوف

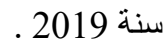

مارينا إيفانوفنا تسفيتايفا، شاعرة روسية سوفييتية ولدت سنة 1892 و توفيت سنة 1941. من أشهر قصائدها : (قصائد عن موسكو) و ( الأرق) عز الدين الثنتوف ،أكاديمي و ناقد مغربي ـ حاصل على جائزة الأطلس الكبير لترجمة لهذا الكتاب (كتابة الفاجعة)

موريس بلانشو. كتابة الفاجعة. ترجمة عز الدين الشنتوف ـ 2018 .دار توبقال ـ ص ص 76 ـ

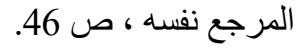

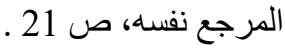

طه عبد الرحمان. في أصول الحوار و تجديد علم الكلام .بيروت ـ المركز الثقافي العربي. 2000. ص 93.

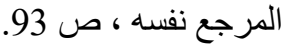

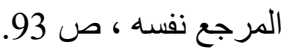

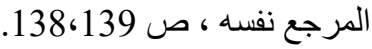




$$
\begin{aligned}
& \text { محاضرة الدكتور حانم باسل، كلية الآداب و العلوم الانسانية بتطوان، } 2018 .
\end{aligned}
$$

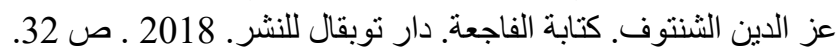

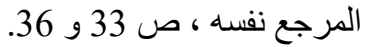

$$
\begin{aligned}
& \text { طه عبد الرحمان. في أصول الحوار و تجديد علم الكر الكلام .بيروت ـ المركز الثقافي العربي. 2000. ص } 139 .
\end{aligned}
$$

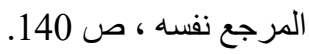

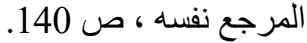

اقتطفت هذه العبارة من مؤلف جماعي بعنوان جاك ديريدا ،فيلسوف الهوامش ،تأملات في التفكيك و الكتابة و السياسة. تحرير : محمد

$$
\text { بكزي. 2017. }
$$

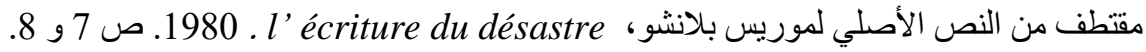

مقتطف من الترجمة الانجليزية لآن سموك، 1995. The Writing of The Disaster ص صن 1.

مقتطف من الترجمة العربية لعز الدين الثنتوف، كتابة الفاجعة . 2018 ص 51. سورة آل عمران ، الآية 190.

اقتطفت هذه العبارة من مؤلف جماعي بلعي بعنوان جاك ديريدا ،فيلسوف الهوامش ،تأملات في التفكيك و الكتابة و السياسة. تحرير : محمد

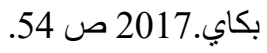$$
\text { جالك، ديريدا. الكتابة و الاختلاف ـ } 1967 \text {. } 19017 .
$$

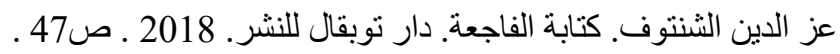

أقتبست هذه العبارة من محاضرة عز الدة الدين الشنتوف دارئ عند تقديمه لهذا الكتاب سنة 2018.

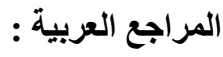

$$
\begin{aligned}
& \text { الادريسي،مزوار. (2019) ـ كتابة الفاجعة : في نقل التلعثم. العربي الجديد .عدد 13مارس 2019. الكويت. }
\end{aligned}
$$

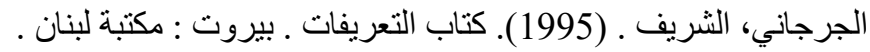

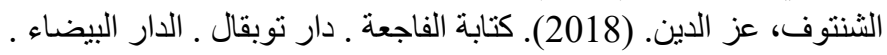

$$
\begin{aligned}
& \text { (5بد الرحمان، طه. (2000). في أصول الحوار و تجديد علم الكلام ـ بيروت : المركز الثقاء الثقافي العربي. }
\end{aligned}
$$

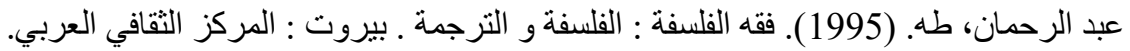

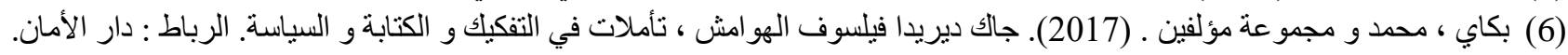

Blanchot, M. (1980) . L’écriture du désastre. Gallimard.

Smock, A . (1995) .The writing of the disaster.University of Nebraska Press. 